\title{
Effects of Filament Diameter Tolerances in Fused Filament Fabrication
}

\author{
Carolina Cardona, Abigail H. Curdes, and Aaron J. Isaacs, Purdue School of Engineering and Technology, \\ Indiana University - Purdue University Indianapolis
}

Faculty Mentor: Dr. Andres Tovar

\section{ABSTRACT}

Fused filament fabrication (FFF) is one of the most popular additive manufacturing (3D printing) technologies due to the growing availability of low-cost desktop 3D printers and the relatively low cost of the thermoplastic filament used in the 3D printing process. Commercial filament suppliers, 3D printer manufacturers, and end-users regard filament diameter tolerance as an important indicator of the 3D printing quality. Irregular filament diameter affects the flow rate during the filament extrusion, which causes poor surface quality, extruder jams, irregular gaps in-between individual extrusions, and/or excessive overlap, which eventually results in failed 3D prints. Despite the important role of the diameter consistency in the FFF process, few studies have addressed the required tolerance level to achieve highest 3D printing quality. The objective of this work is to develop the testing methods to measure the filament tolerance and control the filament fabrication process. A pelletbased extruder is utilized to fabricate acrylonitrile butadiene styrene (ABS) filament using a nozzle of $1.75 \mathrm{~mm}$ in diameter. Temperature and extrusion rate are controlled parameters. An optical comparator and an array of digital calipers are used to measure the filament diameter. The results demonstrate that it is possible to achieve high diameter consistency and low tolerances $(0.01 \mathrm{~mm})$ at low extrusion temperature $\left(180^{\circ} \mathrm{C}\right)$ and low extrusion rate $(10 \mathrm{in} / \mathrm{min})$.

\section{KEYWORDS : acrylonitrile butadiene styrene, RepRap printers, fused filament fabrication, fused deposition modeling, inconsistent extrusion, Raster}

\section{INTRODUCTION}

$\mathrm{F}$ used deposition modeling is a type of additive manufacturing. According to Griffey (2014), "fused deposition modeling is what most people understand to be 3 -D printing, as this technology is by far the most common and in many ways the simplest of the possibilities [of additive manufacturing].” Fused filament fabrication (FFF) works the same way as fused deposition modeling; by laying down molten strings of plastic (in our case ABS) from the nozzle of the printer's hot end onto each other, the molten plastic fuses together to build parts. Parts begin as computer models, which are converted into stereolithography files for printing. When using a RepRap printer, the layers are laid down according to the Cartesian coordinates $(\mathrm{x}, \mathrm{y}, \mathrm{z})$ and the print head is programmed to move with relation to the build plate where the flat surface layers of the object are deposited on. The RepRap Prusa models used in this project print layers from the build plate vertically upwards. An advantage of using a RepRap printer is that a user can control more variables and that these printers can reproduce the parts for themselves by printing them.

With custom programming, users can control certain variables that affect the overall print. One such variable is the layer height. Layer height is the FFF equivalent of a printed page's dots per inch as it determines the resolution of the print; a smaller layer height creates a smoother finished product but it also takes a significant amount of time longer to print such high resolution objects (Griffey, 2014). A common theme in the variables users try to control is one that allows for higher quality prints. 3D printer users have to adjust to a number of issues that can cause prints to be incorrect, or fail. An aim of this research is to determine what makes a print of quality and if the filament's geometric and dimensional tolerances have a part in affecting the quality of finished prints.

Hernandez (2015) suggests that "differences in material properties across manufacturers and even across different material lots from the same manufacturer can result in very different printing results, requiring user intervention to refine several printer parameters until usable prints are achieved." 3D printing software will extrude a certain volume based on the filament diameter, diameter of extrusion nozzle, and the extrusion flow rate. When printing, an extruder wheel turns and pushes some selected volume of plastic down the hot end. If there are any irregularities in the filament being fed to the nozzle, the printer will not compensate for the difference and will continue printing. This is what is referred to as "inconsistent extrusion." The printer is extruding based on a certain theoretical amount already determined in the file and will not adjust the extrusion to fix any unexpected variations of the filament during printing. "Once a given layer is printed, the build platform lowers by the distance equal to the slice layer thickness. With the lowered build platform, the next layer is built on top of the previous layer. This process continues until the build is complete" (Rishi, 2013). This distance does not undergo any automatic adjustments if any imperfections were to occur with the amount of material flowing out of the nozzle.

A filament diameter that is inconsistent can cause serious complications during extrusion. An unexpected small diameter will cause the mechanism to not be able to grip the filament because of the lack of tension, causing extruder 
failure since no plastic will melt for impression. Conversely, if the filament has a sudden increase in diameter, the plastic will not be able to fit in the opening of the nozzle and melt properly. The gold standard across the industry for filament tolerance is $+/-0.05 \mathrm{~mm}$ (or acceptable deviation of $0.1 \mathrm{~mm}$ ) and a typical spool of $1 \mathrm{~kg} \mathrm{ABS}$ is approximately $1350 \mathrm{ft}$. Maintaining a tolerance lower than the current industry standard with consistency throughout the entire length of the filament would be challenging during manufacturing, which is why there is grounds for more research in this area.

It remains debatable if minor changes within tolerance can affect an extrusion. During extrusion, as explained previously, the filament is heated and melted for impression. The imperfections of the filament that can affect printing are those that occur right when the material is being discharged from the nozzle. Roundness, as a matter of fact, is not even a major factor considered. The only importance of it is that any angle printed is within diameter tolerance. Roundness is not used as a key evaluation in our project. Consistency is more important for our research, and tolerances will become our main priority when evaluating filaments.

\section{METHODOLOGY}

Ong (1994) finds that "conventional tolerances allow the designer to specify the desirable surface condition and size of a part feature within an upper and a lower limit. The advantages of this tolerancing method are that it is simple to use and part conformance can be easily verified using direct measurement tools such as a caliper and a surface indicator." For our project we have designed the following objectives and outlined how we will address them.

\subsection{Filament Analysis}

Firstly, we need to specifically understand the filament. We will need to approach the outcomes of filament extrusion, like filament differences, or "air pockets" shown in the Results section. The purpose of this is to identify causes of variations in the material and prevent imperfections during extrusion. Controlled parameters, such as feed rate and temperature, will be studied as possible causes. Distinct adjustments of these parameters should be analyzed to determine the optimum settings for the filament to obtain the consistency desired. Thus, we will improve the potential of our extrusion and eventually narrow tolerance values.

\subsection{Optimization of Extruding Process}

Optimizations will include the improvements in the feed rate, pressure, and temperature during extrusion, following the conclusions obtained from the filament analysis portion. Differences in sizes matter when purchasing a filament and this should be addressed to optimize the accuracy of the filament quality when selecting dimensions. Deciding optimal processing parameters is the basis of this objective. We will have to manipulate controlled parameters to identify their effects and select ideal settings for consistent filament. Control variables will be selected for comparison. Prints with common criteria for printing with selected values in the chosen aspect will be compared to prints made with our adjustments to determine the difference in quality, accuracy, and raster air gap.

The feed rate threshold will be observed in addition to this process. Research has shown that manipulating feed rate and nozzle height can impact volumetric flow rate. This involves affecting surface quality, layer height, and bead width. What this means is if the 3D printer is "aware" of the geometrical and dimensional properties of filament before the filament enters the heated nozzle, past research has shown it is possible to adjust parameters to compensate for these irregularities (Rishi, 2013). However, future research will be needed to determine the significance of a closed loop feedback system on surface quality and part accuracy (Rishi, 2013).

\subsection{Final Adjustments}

If we were to come across the situation where the manipulation of aspects within the printer may not be sufficient to obtain the quality of print desired, we will then add an additional process after extrusion, referring to techniques like cooling down the filament faster, setting the product in a "bath" of water, or adding a chemical to allow the filament material to set. This serves to correct imperfections still present after extrusion.

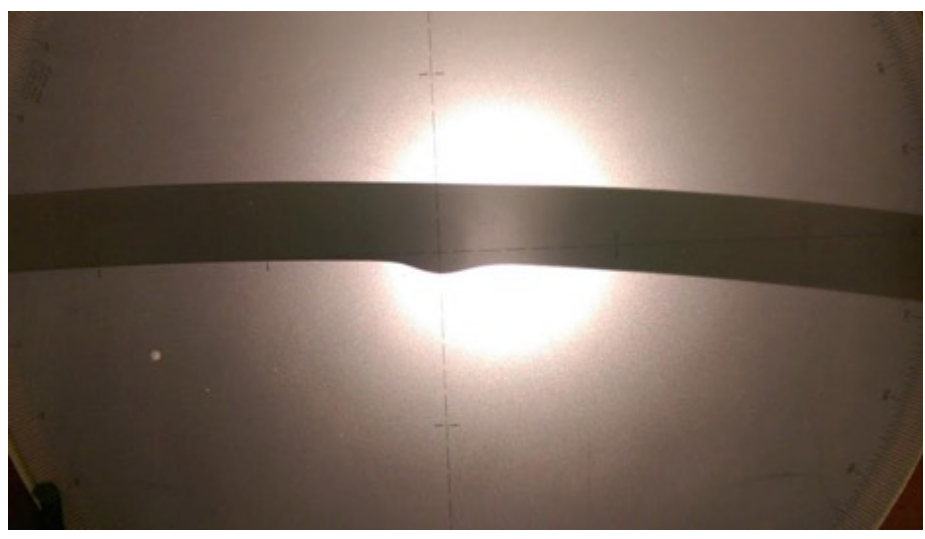

Figure 1. Optical Comparator - Air bubble in purchased filament.

\section{RESULTS}

During the process of evaluating filament, imperfections (see Figure 1) were found that prove the need of 3-axis to monitor filament diameter. It is necessary to develop a way to detect imperfections beyond filament diameter tolerances to maintain the filament within the acceptable ranges. This is necessary to maintain the quality of the print and prevent unwanted results, previously identified as inconsistent extrusions.

Without any measuring instrument, imperfections (see Figure 2) can be difficult to identify. Also, without 3-axis monitoring, any differences that are one-sided can be out of sight once the filament is rotated. Ideally, filament should maintain a consistent diameter throughout the entire spool. In reality, during manufacturing, an absolutely consistent diameter value throughout an entire filament is not achievable, which is why a tolerance has to be defined. Diameter can be 
changed by altering temperature, nozzle diameter, or by pulling the filament as it is extruded.

The orientation of the extrusion device also affects the resulting filament. When the filament does not cool quickly enough, it is still malleable enough to misshape or curl back on itself. Those who extrude filament have documented a preference for angled extrusion, vertically mounted extrusion, and horizontal extrusion with the use of a pulley to pull the filament into a spool. Preferences though, do not provide enough evidence to support whether all methods are acceptable or whether some are more beneficial to end product quality.

A pellet-based extruder is utilized to fabricate acrylonitrile butadiene styrene (ABS) filament using a nozzle of $1.75 \mathrm{~mm}$ in diameter. This nozzle diameter has remained the same throughout this project. Temperature and extrusion rate are other controlled parameters. In our testing of different temperatures, we determined that $174{ }^{\circ} \mathrm{C}$ up to $180{ }^{\circ} \mathrm{C}$, depending on room temperature, successfully extruded filament of the desired diameter. An optical comparator and an array of digital calipers are used to measure the filament diameter currently. The results demonstrate that it is possible to achieve high diameter consistency and low tolerances $(0.01 \mathrm{~mm})$ at low extrusion temperature $\left(180{ }^{\circ} \mathrm{C}\right)$ and low extrusion rate $(10 \mathrm{in} / \mathrm{min})$.

\section{CONCLUSION}

Our significant findings have been building a filament extruder and categorizing the differences between manufactured, massproduced filament, and filament we have extruded from ABS pellets. As presented by the objectives and results, the project is still in process of collecting significant evidence for future research routes to be determined. This project's experience with filament extrusion has shown a need for possibly producing a second extruder to modify for use in the continuation of the project. With the manipulation of certain variables (thus far temperature and orientation of the extruder), we have already seen the effect variable control has on final filament diameter. As stated in the results, there is a varying temperature at which to extrude and this affects the resulting diameter for those producing filament. The understanding of the filament extrusion process is key to manipulating such variables and producing results. There are a great number of components to define in this continuing process. Some of these components are how to specifically characterize a good quality filament, how to achieve and produce good quality filament with consistency, and whether a quality filament improves the quality of a print. In many cases the materials in production do affect resulting products, but as far as 3D printing there is more complete evidence to be found to prove or to disprove this idea. With filament quality there are a multitude of characteristics (i.e., tensile strength) that have the potential to affect the overall quality but which ones do with significance is to be determined still. These are all fields with a necessity of further investigation and a process to follow. It requires us to understand filament to a certain extent in order to be able to quantify the quality of it. Research has shown that manipulating feed rate and nozzle

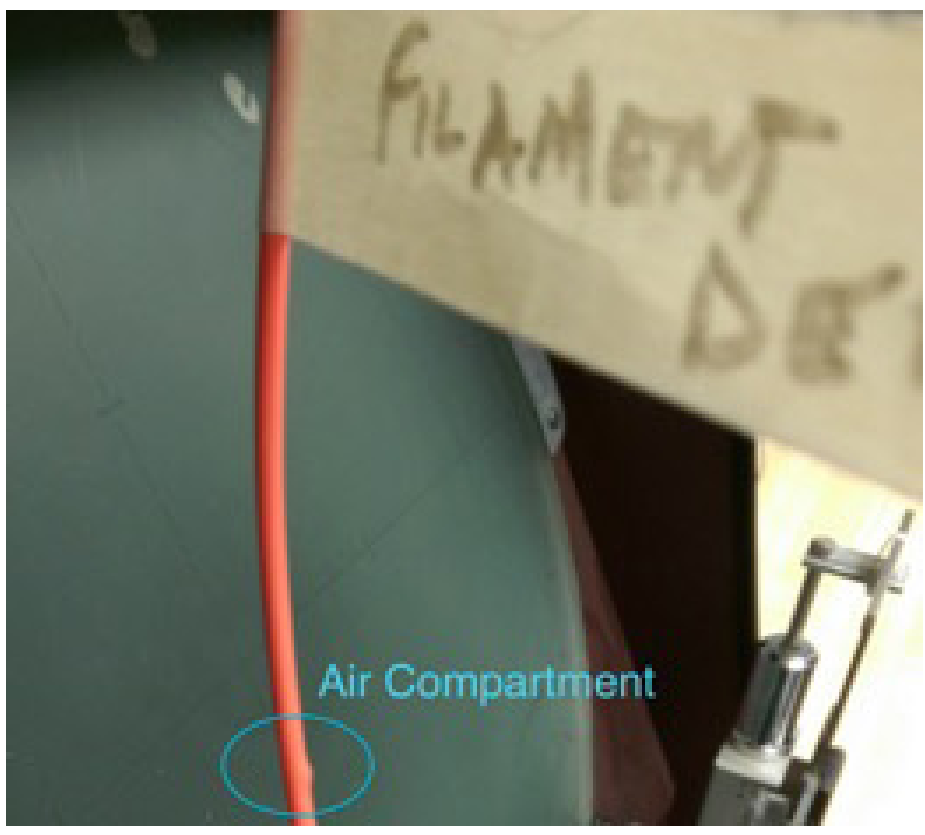

Figure 2. Filament imperfection.

height can impact volumetric flow rate. Parameters will further be established by this project to fulfill these goals.

\section{AUTHOR INFORMATION}

All correspondence should be sent to the first author: cardonca@iupui.edu.

\section{ACKNOWLEDGMENTS}

The authors would like to sincerely thank the extremely helpful collaboration of Carl Marko and Michael Gollub. All their expertise has been of great guidance during this process. They would also like to thank the IUPUI Center for Research and Learning for supporting and facilitating their research and the research of others on campus. Lastly, the authors would like to thank Dr. Andres Tovar for his mentorship and support in the execution of this research project.

\section{REFERENCES}

Bellehumeur, C., Li, L., Sun, Q., \& Gu, P. (2004). Modeling of bond formation between polymer filaments in the fused deposition modeling process. Journal of Manufacturing Processes, 6(2), 170-178.

Griffey, J. (2014). The types of 3-D printing. Library Technology Reports, 50(5), 8-12.

Hernandez, D. D. (2015). Factors affecting dimensional precision of consumer 3D printing. International Journal of Aviation, Aeronautics, and Aerospace, 2(4).

Perez, A. R. T., Roberson, D. A., \& Wicker, R. B. (2014). Fracture surface analysis of 3D-printed tensile specimens of novel ABS-based materials. Journal of Failure Analysis and Prevention, 14(3), 343-353. 
Ong, J. B. (1994). Geometric modeling of manufacturing processes variations for model-based tolerance analysis (Doctoral dissertation). Retrieved from Virginia Tech Electronic Theses and Dissertations: http://vtechworks. lib.vt.edu/handle/10919/27533?show=full

Rishi, O. (2013). Feed rate effects in freeform filament extrusion (Master's thesis). Retrieved from Rochester Institute of Technology: http://scholarworks.rit.edu/ 